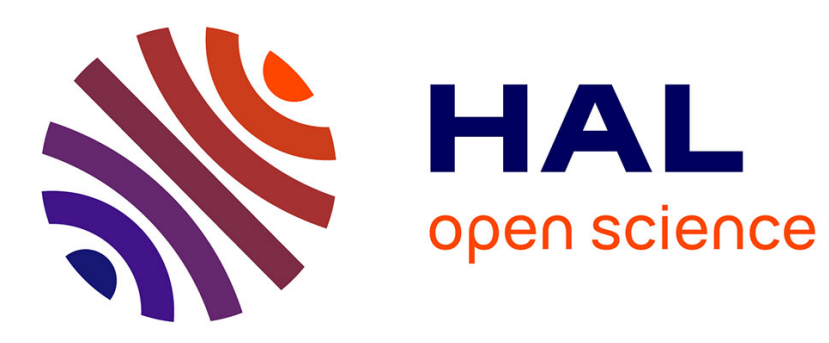

\title{
Boundary value problems and convolutional systems over rings of ultradistributions
}

Hugues Mounier, Joachim Rudolph, Frank Woittennek

\section{To cite this version:}

Hugues Mounier, Joachim Rudolph, Frank Woittennek. Boundary value problems and convolutional systems over rings of ultradistributions. Jean Levine,Philippe Müllhaupt. Advances in the Theory of Control, Signals and Systems with Physical Modeling, Springer, pp.179-188, 2010, Lecture Notes in Control and Information Sciences. hal-00526150

\section{HAL Id: hal-00526150 https://hal.science/hal-00526150}

Submitted on 13 Oct 2010

HAL is a multi-disciplinary open access archive for the deposit and dissemination of scientific research documents, whether they are published or not. The documents may come from teaching and research institutions in France or abroad, or from public or private research centers.
L'archive ouverte pluridisciplinaire HAL, est destinée au dépôt et à la diffusion de documents scientifiques de niveau recherche, publiés ou non, émanant des établissements d'enseignement et de recherche français ou étrangers, des laboratoires publics ou privés. 


\title{
Boundary Value Problems and Convolutional Systems over Rings of Ultradistributions
}

\author{
Hugues Mounier, Joachim Rudolph, and Frank Woittennek
}

\begin{abstract}
One dimensional boundary value problems with lumped controls are considered. Such systems can be modeled as modules over a ring of Beurling ultradistributions with compact support. This ring appears naturally from a corresponding Cauchy problem. The heat equation with different boundary conditions serves for illustration.
\end{abstract}

\section{Introduction}

The design of feedforward and feedback control for finite dimensional systems and delay systems is largely simplified by flatness based control, respectively freeness. This has been shown in numerous academic case studies and industrial applications. A central part in the control design (the importance of which has often been underestimated) is trajectory planning.

It is particularly useful for distributed parameter systems with lumped control inputs, a class of systems the models of which include partial differential equations. In the linear case, as for delay systems, a module-theoretic framework has been established, and the trajectory planning is based on the use of a module basis, which plays a role similar to the one of a flat output in finite-dimensional flat systems.

Examples of distributed parameter systems that have been studied are heat conductors, elastic piezo-beams and plates, elastic robot arms, ropes, electric lines,

Hugues Mounier

Laboratoire des Signaux et Systèmes, Supélec, 3, rue Joliot Curie, 91192 Gif-sur-Yvette, France

Joachim Rudolph

Lehrstuhl für Systemtheorie und Regelungstechnik, Universität des Saarlandes, Campus A5 1, 66123 Saarbrücken, Germany, e-mail: j.rudolph@1sr.uni-saarland.de

Frank Woittennek

Institut für Regelungs- und Steuerungstheorie, TU Dresden, 01062 Dresden, Germany, e-mail: frank.woittennek@tu-dresden.de 
tubular chemical reactors, and heat exchangers (see, e.g., [16, 17]). Although many of the problems considered are linear with fixed boundary, some nonlinear and free boundary value problems have been solved, too.

Here, based on the example of the linear heat equation the choice of the ring used to represent the system as a module is further discussed. It is shown that a suitable ring is $\mathscr{R}=\mathbb{C}\left(\partial_{t}\right)[\mathfrak{S}] \cap \mathscr{E}^{\prime *}$, where $\partial_{t}$ stands for time derivation, $\mathfrak{S}$ is a collection of spatially dependant hyperbolic functions, and $\mathscr{E}^{\prime} *$ is a ring of Beurling ultradistributions.

\section{Motivating Example: the Heat Equation}

The one dimensional heat equation might be viewed as one of the simplest problems of the class considered in the sequel. It will, therefore, be used for motivation. Moreover, this discussion is based on elementary calculations, which allow one to capture the idea of the approach without entering into deeper mathematical considerations.

Consider the system

$$
\begin{aligned}
& \partial_{x}^{2} w(x, t)=\partial_{t} w(x, t), \quad x \in[0,1], t \in \mathbb{R} \\
& \partial_{x} w(0, t)=0, \quad w(1, t)=u(t)
\end{aligned}
$$

with homogeneous initial conditions. These equations model the heat conduction in a rod of unit length, where $w(x, t)$ denotes the temperature at the point $x$ at time $t$. The first boundary condition means that there is no heat flux at $x=0$, the second one means that the temperature at $x=1$ is considered as a control input $u(t)$.

\subsection{Symbolic Viewpoint}

Use the Laplace transform w.r.t. $t$ to obtain

$$
s \widehat{w}(x, s)=\partial_{x}^{2} \widehat{w}(x, s)
$$

from (1a). (Mikusiński's oprational calculus would lead to similar formulae.) The characteristic equation associated with (2) reads $\zeta^{2}-s=0$, i.e. $\zeta= \pm \sqrt{s}$, and the general solution of (1a) can, thus, be written as $\widehat{w}(x, s)=e^{x \sqrt{s}} \gamma_{1}(s)+e^{-x \sqrt{s}} \gamma_{2}(s)$ or

$$
\widehat{w}(x, s)=\cosh (x \sqrt{s}) \lambda_{1}(s)+\frac{\sinh (x \sqrt{s})}{\sqrt{s}} \lambda_{2}(s) .
$$

The second formulation is easier to handle, because with

$$
\widehat{C}_{0}(x)=\cosh (x \sqrt{s}), \quad \widehat{C}_{1}(x)=\frac{\sinh (x \sqrt{s})}{\sqrt{s}}
$$


one has the relations $\partial_{x} \widehat{C}_{0}(x)=s \widehat{C}_{1}(x), \partial_{x} \widehat{C}_{1}(x)=\widehat{C}_{0}(x)$. Furthermore, as $\widehat{C}_{0}(0)=$ 1 and $\widehat{C}_{1}(0)=0$, the parameters $\lambda_{1}$ and $\lambda_{2}$ admit a direct interpretation through $\lambda_{1}(s)=\hat{w}(0, s)$ and $\lambda_{2}(s)=\partial_{x} \widehat{w}(0, s)$. The general form of the solution and its first derivative can thus be written

$$
\begin{aligned}
\widehat{w}(x, s) & =\widehat{C}_{0}(x) \lambda_{1}(s)+\widehat{C}_{1}(x) \lambda_{2}(s) \\
\partial_{x} \widehat{w}(x, s) & =s \widehat{C}_{1}(x) \lambda_{1}(s)+\widehat{C}_{0}(x) \lambda_{2}(s) .
\end{aligned}
$$

The boundary conditions (1b) yield

$$
\lambda_{2}(s)=0, \quad \widehat{C}_{0}(1) \lambda_{1}(s)=\widehat{u}(s),
$$

and the equation $\cosh (\sqrt{s}) \widehat{w}(x, s)=\cosh (x \sqrt{s}) \widehat{u}(s)$, or

$$
\widehat{C}_{0}(1) \widehat{w}(x, s)=\widehat{C}_{0}(x) \widehat{u}(s) .
$$

As a result one has a parametrization in $\lambda_{1}(s)$ :

$$
\begin{aligned}
\widehat{u}(s) & =\widehat{C}_{0}(1) \lambda_{1}(s) \\
\widehat{w}(x, s) & =\widehat{C}_{0}(x) \lambda_{1}(s) .
\end{aligned}
$$

The free parameter $\lambda_{1}$ may, therefore, be considered as a flat or basic output. In a module theoretic framework on an appropriate ring (to be defined) it would form a basis of a corresponding free module.

Formally, write $\cosh (\sqrt{s})=\sum_{i \geqslant 0} s^{i} /((2 i) !)$, and introduce $\omega(t)=w(0, t)$ to denote the function corresponding to $\lambda_{1}$ in the time domain. Then, in the time domain

$$
w(x, t)=\sum_{i \geqslant 0} \frac{x^{2 i}}{(2 i) !} \omega^{(i)}(t), \quad u(t)=\sum_{i \geqslant 0} \frac{1}{(2 i) !} \omega^{(i)}(t) .
$$

Convergence of the above series can be shown (see, e.g., [6, 11, 12]) provided $t \mapsto$ $\omega(t)$ is a Beurling ultradifferentiable function of Gevrey order 2 (cf. the app.).

\subsection{Temporal Viewpoint}

A different look on the problem is based on a Cauchy-Kowaleski form of the system:

$$
\begin{aligned}
& \partial_{x}^{2} w(x, t)=\partial_{t} w(x, t), \quad x \in[0,1], t \in[0, \infty[ \\
& \partial_{x} w(0, t)=0, \quad w(0, t)=\omega(t),
\end{aligned}
$$

which allows one to search for a formal solution

$$
w(x, t)=\sum_{i \geqslant 0} a_{i}(t) \frac{x^{i}}{i !}
$$


where the functions $a_{i}$ are infinitely differentiable. A formal check based upon (7) gives $a_{i+2}(t)=\dot{a}_{i}(t), i \geqslant 0, a_{1}(t)=0, a_{0}(t)=\omega(t)$. Thus, for $i \geqslant 0$, one has $a_{2 i}(t)=$ $\omega^{(i)}(t), a_{2 i+1}(t)=0$, which implies (6).

\section{Module Theoretic Formulation over Appropriate Rings}

Generalizing the ideas of the introductory example, this section describes how boundary value problems can be reformulated as linear systems of equations over rings of ultradistributions. These equations serve as the defining relations for the module representing the system under consideration. The question of the appropriate choice of the coefficient rings of this module is brought up because its particular choice may play an important rhole in whether the system module is free. The latter property essentially simplifies trajectory planning and control design.

\subsection{Class of models considered}

In order to keep the exposition simple, in the sequel the following particular class of systems, with distributed variables $w_{1}, \ldots, w_{l}$ and lumped variables $u=\left(u_{1}, \ldots, u_{m}\right)$ is considered:

$$
\begin{aligned}
\partial_{x} w_{i}=A_{i} w_{i}+B_{i} u, \quad w_{i}: \Omega_{i} \rightarrow \mathscr{F}^{p}, \quad u \in \mathscr{F}^{m} \\
A_{i} \in\left(\mathbb{R}\left[\partial_{t}\right]\right)^{p_{i} \times p_{i}}, \quad B_{i} \in\left(\mathbb{R}\left[\partial_{t}\right]\right)^{p_{i} \times m}, \quad i \in\{1, \ldots, l\}
\end{aligned}
$$

where $\mathscr{F}$ represents an appropriate space $\mathscr{E}^{*}(\mathbb{R})$ of smooth functions or (ultra-) distributions $\mathscr{D}^{\prime}(\mathbb{R})$ to be specified in Sect. 3.2 below. The intervals $\Omega_{1}, \ldots, \Omega_{l}$ are open neighborhoods of $\tilde{\Omega}_{i}=\left[x_{i, 0}, x_{i, 1}\right]$. Without loss of generality, assume $x_{i, 0}=0$.

A key hypothesis will be the following: The characteristic polynomials of the matrices $A_{1}, \ldots, A_{l}$ can be written

$$
P_{i}(\lambda):=\operatorname{det}\left(\lambda I-A_{i}\right)=\sum_{v=0}^{p_{i}} a_{i, v} \lambda^{v}, \quad a_{i, v}=\sum_{\mu \leq p_{i}-v} a_{i, v, \mu} \partial_{t}^{\mu}
$$

with $a_{i, v, \mu} \in \mathbb{R}, a_{i, p_{i}, 0}=1$. Moreover, their principal parts $\sum_{\mu+v=p_{i}} a_{i, v, \mu} \partial_{t}^{\mu} \lambda^{v}$ are hyperbolic w.r.t. the time $t$, i.e., the roots of $\sum_{\mu+v=p_{i}} a_{i, v, \mu} \lambda^{v}$ are real.

The models are completed by boundary conditions

$$
\sum_{i=1}^{l} L_{i} w_{i}(0)+R_{i} w_{i}\left(\ell_{i}\right)+D u=0
$$

with $D \in\left(\mathbb{R}\left[\partial_{t}\right]\right)^{q \times m}$ and $L_{i}, R_{i} \in\left(\mathbb{R}\left[\partial_{t}\right]\right)^{q \times p_{i}}$. 
Remark 1. Note that the above assumptions apply to a large class of spatially onedimensional boundary controlled evolution equations, including Euler-Bernoulli or Timoshenko beam equations, more general parabolic diffusion-reaction-convection equations, damped and undamped wave-equations etc. An exception are the models of internally damped mechanical systems.

Example 1. Consider an example similar to (1). The model is given by

$$
\begin{aligned}
& \partial_{x}^{2} w(x, t)=\partial_{t} w(x, t), \quad x \in[0, \ell], t \in[0,+\infty[ \\
& \partial_{x} w(0, t)=0, \quad \partial_{x} w(\ell, t)=u(t),
\end{aligned}
$$

which may be rewritten in the form $(8 \mathrm{a}),(8 \mathrm{c})$ as

$$
\begin{aligned}
\partial_{x}\left(\begin{array}{c}
w(x, t) \\
\partial_{x} w(x, t)
\end{array}\right) & =\left(\begin{array}{ll}
0 & 1 \\
\partial_{t} & 0
\end{array}\right)\left(\begin{array}{c}
w(x, t) \\
\partial_{x} w(x, t)
\end{array}\right) \\
\left(\begin{array}{ll}
0 & 1 \\
0 & 0
\end{array}\right)\left(\begin{array}{c}
w(0, t) \\
\partial_{x} w(0, t)
\end{array}\right)+\left(\begin{array}{ll}
0 & 0 \\
0 & 1
\end{array}\right)\left(\begin{array}{c}
w(\ell, t) \\
\partial_{x} w(\ell, t)
\end{array}\right) & =\left(\begin{array}{l}
0 \\
1
\end{array}\right) u(t) .
\end{aligned}
$$

The characteristic polynomial $P(\lambda)=\lambda^{2}-\partial_{t}$ of the coefficient matrix in (10a) has the principal part $\lambda^{2}$ which is clearly hyperbolic w.r.t. the time axis.

\subsection{Solution of the Cauchy Problem}

Some properties of the solution of the Cauchy problem (8a) with initial conditions given at $x=\xi$, i.e.

$$
\partial_{x} w=A w+B u, \quad w(\xi)=w \xi
$$

with $A \in\left(\mathbb{R}\left[\partial_{t}\right]\right)^{p \times p}, B \in\left(\mathbb{R}\left[\partial_{t}\right]\right)^{p \times q}$ as assumed in the previous section for $A_{i}, B_{i}$, will be used. The notation of the previous section is used in what follows, dropping the index $i \in\{1, \ldots, l\}$.

Choose $^{1} \mathscr{E}^{*}(\mathbb{R})=\mathscr{E}^{(p /(p-1))}(\mathbb{R})\left(\right.$ resp. $\left.\mathscr{D}^{\prime}(\mathbb{R})=\mathscr{D}^{\prime}(p /(p-1))(\mathbb{R})\right)$ which corresponds to Beurling ultradifferentiable functions (resp. ultradistributions) of Gevrey order $p /(p-1)$ introduced in the appendix.

Consider the initial value problem

$$
P\left(\partial_{x}\right) v(x)=0, \quad\left(\partial_{x}^{j} v\right)(0)=v_{j} \in \mathscr{F}, \quad j=0, \ldots, p-1
$$

associated with the characteristic polynomial

$$
P(\lambda):=\operatorname{det}(\lambda I-A)=\sum_{j=0}^{p} a_{j} \lambda^{j}, \quad a_{j}=\sum_{\mu \leq p-j} a_{j, \mu} \partial_{t}^{\mu} .
$$

\footnotetext{
${ }^{1}$ Depending on the particular p.d.e. under consideration, choosing larger spaces $\mathscr{E}^{*}$ of smooth functions and smaller spaces $\mathscr{D}^{*}$ of ultradistributions and even distributions may be possible.
} 
Conformal with [8, Thrm. 12.5.6] or [15, Thrm 2.5.2, Prop. 2.5.6] the initial value problem (12) has a unique solution. This solution may be written as

$$
v(x)=\sum_{j=0}^{p-1} C_{j}(x) v_{j}
$$

where juxtaposition of symbols means convolution and $C_{0}, \ldots, C_{p-1}$ are smooth functions ${ }^{2}$ mapping $\Omega$ to the space of compactly supported Beurling ultradistributions $\mathscr{E}^{\prime} *(\mathbb{R}):=\mathscr{E}^{\prime}(p /(p-1))(\mathbb{R})$ of Gevrey order $p /(p-1)$. The functions $C_{0}, \ldots, C_{p-1}$ satisfy $(k, j \in\{0, \ldots, p-1\})$

$$
\partial_{x}^{k} C_{j}(0)= \begin{cases}1, & k=j \\ 0, & k \neq j\end{cases}
$$

and

$$
\partial_{x} C_{j}=C_{j-1}-a_{j} C_{p-1}, \quad j=1, \ldots, p-1, \quad \partial_{x} C_{0}=-a_{0} C_{p-1} .
$$

With these preparatory steps, the unique solution $x \mapsto \Phi(x, \xi)$ of the initial value problem (11) can be expressed as

$$
w(x)=\Phi(x, \xi) w_{\xi}+\Psi(x, \xi) u .
$$

Therein, $\Phi(x, \xi) \in \mathscr{E}^{\prime} *(\mathbb{R})^{p \times p}$ and $\Psi(x, \xi) \in \mathscr{E}^{\prime *}(\mathbb{R})^{p \times m}$ are given by

$$
\Phi(x, \xi)=\sum_{j=0}^{p-1} A^{j} C_{j}(x-\xi), \quad \Psi(x, \xi)=\int_{\xi}^{x} \Phi(x, \zeta) d \zeta B .
$$

That (15) with the matrices given in (16) is indeed a solution of (11) can be checked by plugging it into the p.d.e. in (11) and then employing (14) in combination with the Cayley-Hamilton theorem. Moreover, observe that $\Psi(\xi, \xi)=0$ while $\Phi(\xi, \xi)$ is the identity. As a consequence, the restriction of $x \mapsto w(x)$ to $x=\xi$ indeed equals $w_{\xi}$.

Uniqueness of the solution (15) can be led back to the uniqueness of the scalar problem (12). To this end assume the existence of two different solutions of (11) which, by linearity, implies the existence of a non-zero solution of the homogeneous p.d.e. $\partial_{x} \tilde{w}(x)=A \tilde{w}(x)$ with data $\tilde{w}(\xi)=0$. Differentiating this latter differential equation $p-1$ times w.r.t. $x$ and using the Cayley-Hamilton theorem, one observes that all components of $\tilde{w}$ satisfy (12) with zero data $\tilde{w}(\xi)=\cdots=\partial_{x}^{p-1} \tilde{w}(\xi)=0$.

Remark 2. As in the example introduced in sec. 2 the solution of the Cauchy problem (11) can be achieved either by direct computations in the time domain (cf. sec. 2.2) or, alternatively, by means of the Laplace transform (cf. sec. 2.1). According to the classical theory of ordinary differential equations, the solution of the Cauchy

\footnotetext{
${ }^{2}$ A function $C: \Omega \rightarrow \mathscr{E}^{\prime *}$ is called of class $C^{\infty}$ if it defines a map $\mathscr{D}^{*} \rightarrow C^{\infty}(\Omega)$, i.e., for any test function $\varphi \in \mathscr{D}^{*}$ the function $\Omega \ni x \mapsto C(x)[\varphi]$ belongs to $C^{\infty}(\Omega)$. It can be shown that this mapping is continuous.
} 
problem (11) in the Laplace domain always exists even if the characteristic polynomial of $A$ does not satisfy the conditions formulated in section 3.1. However, these conditions are necessary in order to ensure the existence of time-domain interpretations of such solutions as compactly-supported ultradistributions. More specifically, they ensure particular growth bounds (w.r.t. the complex Laplace variable $s$ ) of the partial Laplace transforms $\widehat{C}_{0}(x), \ldots, \widehat{C}_{p-1}(x)$ w.r.t. time of $C_{0}(x), \ldots, C_{p-1}(x)$. These bounds are specified in the appropriate Paley-Wiener theorems for ultradistributions (see, e.g., $[9,10,15])$ and distributions (see, e.g., [7]).

Example 2 (Ex. 1 continued). As $p=2$, for every fixed $x \in \Omega, C_{0}(x), C_{1}(x)$ are ultradistributions of Gevrey order 2 (elements of $\mathscr{E}^{\prime}(2)$ ). Clearly, for this simple example $C_{0}(x), C_{1}(x)$ can be given explicitly: While their Laplace transforms simply correspond to (4), in the time domain one gets for all $v_{0}, v_{1} \in \mathscr{E}^{(2)}(\mathbb{R})$ (cf. (6))

$$
C_{0}(x) v_{0}=\sum_{k=0}^{\infty} \frac{x^{2 k}}{(2 k) !} \partial_{t}^{k} v_{0}, \quad C_{1}(x) v_{1}=\sum_{k=0}^{\infty} \frac{x^{2 k+1}}{(2 k+1) !} \partial_{t}^{k} v_{1} .
$$

According to (15) and (16) the solutions of the (spatial) Cauchy problem with data $w(\xi)=c=\left(c_{1}, c_{2}\right)^{T}$ is given by

$$
w(x)=\Phi(x, \xi) c, \quad \Phi(x, \xi)=\left(\begin{array}{cc}
C_{0}(x-\xi) & C_{1}(x-\xi) \\
\partial_{t} C_{1}(x-\xi) & C_{0}(x-\xi)
\end{array}\right) .
$$

In particular, one has $w(x)=C_{0}(x-\xi) c_{1}+C_{1}(x-\xi) c_{2}$.

\subsection{System Module}

Using the solutions of the initial value problem in the boundary conditions ( $8 \mathrm{c})$, one obtains

$$
w_{i}(x)=\Phi_{i}\left(x, \xi_{i}\right) w_{i}\left(\xi_{i}\right)+\Psi_{i}\left(x, \xi_{i}\right) u, \quad i=1, \ldots, l, \quad P_{\xi} c_{\xi}=0
$$

Here $\xi=\left(\xi_{1}, \ldots, \xi_{l}\right)$ is arbitrary but fixed, $c_{\xi}^{T}=\left(w_{1}^{T}\left(\xi_{1}\right), \ldots, w_{l}^{T}\left(\xi_{l}\right), u^{T}\right), P_{\xi}=$ $\left(P_{\xi, 1}, \ldots, P_{\xi, l+1}\right)$ with

$$
\begin{aligned}
P_{\xi, i} & =L_{i} \Phi_{i}\left(0, \xi_{i}\right)+R_{i} \Phi_{i}\left(\ell_{i}, \xi_{i}\right), \quad i=1, \ldots, l \\
P_{\xi, l+1} & =D+\sum_{i=1}^{l} L_{i} \Psi_{i}\left(0, \xi_{i}\right)+R_{i} \Psi_{i}\left(\ell_{i}, \xi_{i}\right) .
\end{aligned}
$$

The system will be represented by a module generated by $c_{\xi}, u$ with the presentation given in (18) - cf. [4, 3, 2, 13]. The ring of coefficients must contain at least the entries of $\Phi_{i}\left(x, \xi_{i}\right), \Psi_{i}\left(x, \xi_{i}\right), i=1, \ldots, l$, and the entries of $P_{\xi}$, which consist of values of functions $C_{i, j}, j=1, \ldots, p_{i}, i=1, \ldots, l$ from $\mathbb{R}$ in $\mathscr{E}^{\prime} \star$. Moreover, the 
matrices may also contain values of spatial integrals of $C_{i, j}$. A possible choice for the ring of coefficients is, thus, $\mathscr{R}^{I}=\mathbb{C}\left[\partial_{t}, \mathfrak{S}, \mathfrak{S}^{I}\right] \subset \mathscr{E}^{\prime *}$ with

$$
\begin{aligned}
\mathfrak{S} & =\left\{C_{i, j}(x) \mid x \in \mathbb{R} ; i=1, \ldots, l ; j=0, \ldots, p_{i}-1\right\}, \\
\mathfrak{S}^{I} & =\left\{C_{i, j}^{I}(x) \mid x \in \mathbb{R} ; i=1, \ldots, l ; j=0, \ldots, p_{i}-1\right\}
\end{aligned}
$$

and

$$
C_{i, j}^{I}(x)=\int_{0}^{x} C_{i, j}(\zeta) d \zeta, \quad i=1, \ldots, l, \quad j=0, \ldots, p_{i}-1 .
$$

This ring is isomorphic to a subring of $\mathscr{E}^{\prime} *$.

Following $[14,1,5]$, in order to simplify the analysis of the module properties instead of $\mathscr{R}^{I}$, the larger ring $\mathscr{R}=\mathbb{C}\left(\partial_{t}\right)[\mathfrak{S}] \cap \mathscr{E}^{\prime} *$ may be considered.

Definition 1. The convolutional system $\Sigma$ associated with the boundary value problem (8) is the module generated by the components of $c_{\xi}$ and $u$ over $\mathscr{R}$, with the presentation matrix $P_{\xi}$.

One may check that $\Sigma$ is independent of the choice of $\xi$ (cf. [19, Sect. 3.3] and [18, Remark 4]).

Example 3 (Ex. 2 continued). Substituting (17) into the boundary conditions (10b) one obtains $L \Phi(0, \xi) c+R \Phi(\ell, \xi) c-D u=0$ or, even more explicitly,

$$
\left(\begin{array}{ll}
0 & 1 \\
0 & 0
\end{array}\right)\left(\begin{array}{cc}
C_{0}(-\xi) & C_{1}(-\xi) \\
\partial_{t} C_{1}(-\xi) & C_{0}(-\xi)
\end{array}\right) c+\left(\begin{array}{ll}
0 & 0 \\
0 & 1
\end{array}\right)\left(\begin{array}{cc}
C_{0}(\ell-\xi) & C_{1}(\ell-\xi) \\
\partial_{t} C_{1}(\ell-\xi) & C_{0}(\ell-\xi)
\end{array}\right) c-\left(\begin{array}{l}
0 \\
1
\end{array}\right) u=0 .
$$

As a result, one has

$$
\left(\begin{array}{ccc}
-\partial_{t} C_{1}(\xi) & C_{0}(\xi) & 0 \\
\partial_{t} C_{1}(\ell-\xi) & C_{0}(\ell-\xi) & -1
\end{array}\right)\left(\begin{array}{l}
c_{1} \\
c_{2} \\
u
\end{array}\right)=0, \quad w(x)=\Phi(x, \xi)\left(\begin{array}{l}
c_{1} \\
c_{2}
\end{array}\right)
$$

the first equation of which may be written

$$
P_{\xi}\left(\begin{array}{l}
c \\
u
\end{array}\right)=0 \quad \text { with } \quad P_{\xi}=\left(\begin{array}{ccc}
-\partial_{t} C_{1}(\xi) & C_{0}(\xi) & 0 \\
\partial_{t} C_{1}(\ell-\xi) & C_{0}(\ell-\xi) & -1
\end{array}\right)
$$

Thus, the convolutional system $\Sigma$ associated with the boundary value problem (10) is the module generated by $c_{1}, c_{2}$, and $u$ over $\mathscr{R}=\mathbb{C}\left(\partial_{t}\right)\left[\left\{C_{0}(x), C_{1}(x) \mid x \in \mathbb{R}\right\}\right] \cap \mathscr{E}^{\prime} *$, with the above defined presentation matrix $P_{\xi}$. Alternatively, instead of starting with a module over $\mathscr{R}$ one may directly pass to $\mathscr{E}^{\prime} *$.

\section{Conclusion}

A ring has been exhibited over which systems of one dimensional boundary controlled distributed parameter systems may be viewed as convolutional systems. It 
appears that this ring is well suited for controllability studies, especially when one is interested in the relations between algebraic and trajectory related controllabillity properties. For a particular subclass of the class of models considered here, it is established in [20], through Bézout ring properties, that torsion freeness and freeness are equivalent over such types of rings for systems in which the p.d.e.'s are of second order only. However, known results for the rings of entire functions of Paley-Wiener type (which are isomorphic to $\mathscr{E}^{\prime} *$ via the Laplace transform) suggest that in some situations it may be advantageous to consider systems over even larger subrings of $\mathscr{E}^{\prime} *$ to obtain similar results.

\section{Appendix: Ultradistributions and Ultradifferentiable Functions}

Some basic definitions about Gevrey functions and the corresponding classes of ultradistributions are recalled here.

Definition 2 (see, e.g. [9],[8, Def. 12.7.3, p. 137]). An infinitely differentiable function $f: \Omega \rightarrow \mathbb{C}$ (with $\Omega \subset \mathbb{R}^{n}$ open) belongs to the small Gevrey class $\mathscr{E}(\alpha)(\Omega)$ (or the space of Beurling ultradifferentiable functions of Gevrey class $\alpha$ ) if for all $M \in \mathbb{R}^{+}$and all compact sets $K \subset \Omega$ there exists $C_{K, M}$ such that

$$
\sup _{t \in \Omega, k \geq 0}\left|\partial_{t}^{(k)} f(t)\right| \leq C_{K, M} M^{k}(k !)^{\alpha} .
$$

A sequence $\left(f_{n}\right), n \in \mathbb{N}, f_{n} \in \mathscr{E}^{(\alpha)}(\Omega)$ converges to $f \in \mathscr{E}^{(\alpha)}(\Omega)$, if for all compact $K \subset \Omega$ and all $M \in \mathbb{R}^{+}$

$$
\lim _{n \rightarrow \infty} \sup _{t \in \Omega, k \geq 0} \frac{\left|\partial_{t}^{(k)}\left(f_{n}(t)-f(t)\right)\right|}{M^{k}(k !)^{\alpha}}=0
$$

The space of compactly supported functions in $\mathscr{E}^{(\alpha)}$ is denoted by $\mathscr{D}^{\alpha}(\Omega)$. A sequence $\left(f_{n}\right), f_{n} \in \mathscr{D}^{(\alpha)}(\Omega), n \in \mathbb{N}$ converges in $\mathscr{D}^{(\alpha)}(\Omega)$ if it converges in $\mathscr{E}^{(\alpha)}(\Omega)$ and, moreover, $\cup_{n \in \mathbb{N}} \operatorname{supp} f_{n}$ is compact. The space $\mathscr{D}^{\prime}(\alpha)(\mathbb{R})\left(\right.$ resp. $\mathscr{E}^{\prime}(\alpha)(\mathbb{R})$ ) of Beurling ultradistributions (resp. Beurling ultradistributions with compact support) of Gevrey order $\alpha$ is the space of linear continuous functionals on $\mathscr{D}^{(\alpha)}(\mathbb{R})$ (resp. $\left.\mathscr{E}^{(\alpha)}(\mathbb{R})\right)$.

The Laplace transform of an ultradistribution $f \in \mathscr{E}^{\prime} *$ is given by $\widehat{f}(s)=f\left(g_{\xi}\right)$ with $g_{s}(t)=\mathrm{e}^{-s t}$. The isomorphism between the two convolution rings of ultradistributions with compact support and their Laplace transforms is given by a PaleyWiener type theorem which can be found in [10]. 


\section{References}

1. Brethé, D., Loiseau, J.J.: A result that could bear fruit for the control of delay-differential systems. In: Proc. 4th IEEE Mediterranean Symp. Control Automation, pp. 168-172. Chania, Greece (1996)

2. Fliess, M., Mounier, H.: Controllability and observability of linear delay systems: an algebraic approach. ESAIM: COCV (Control, Optimisation and Calculus of Variations) 3, 301-314 (1998)

3. Fliess, M., Mounier, H.: Tracking control and $\pi$-freeness of infinite dimensional linear systems. In: G. Picci, D.S. Gilliam (eds.) Dynamical Systems, Control, Coding, Computer Vision, pp. 45-68. Birkhäuser, Basel (1999)

4. Fliess, M., Mounier, H.: An algebraic framework for infinite dimensional linear systems. eSTA (Sciences et Technologies de l'Automatique) 1(1) (2004)

5. Glüsing-Lüerßen, H.: A behavioral approach to delay-differential systems. SIAM J. Control Optim. 35(2), 480-499 (1997)

6. Hill, C.D.: Parabolic equations in one space variable and the non-characteristic Cauchy problem. Communications on Pure and Applied Mathematics XX, 619-633 (1967)

7. Hörmander, L.: The Analysis of Linear Partial Differential Operators I: Distribution Theory and Fourier Analysis, Grundlehren der mathematischen Wissenschaften, vol. 256. SpringerVerlag, Berlin, Heidelberg, New York (1983)

8. Hörmander, L.: The Analysis of Linear Partial Differential Operators II: Differential Operators with Constant Coefficients, Grundlehren der mathematischen Wissenschaften, vol. 257, 2. edn. Springer-Verlag, Berlin, Heidelberg, New York (1990)

9. Komatsu, H.: Ultradistributions. I. Structure theorems and a characterization. J. Fac. Sci. Univ. Tokyo Sect. IA Math. 20, 25-105 (1973)

10. Komatsu, H.: Ultradistributions II. The kernel theorem and ultradistributions with support in a manifold. J. Fac. Sci. Univ. Tokyo Sect. IA Math. 24, 607-628 (1973)

11. Laroche, B., Martin, P., Rouchon, P.: Motion planning for the heat equation. Int. J. Robust and Nonlinear Control 10, 629-643 (2000)

12. Lynch, A.F., Rudolph, J.: Flatness-based boundary control of a class of quasilinear parabolic distributed parameter systems. Internat. J. Control 75(15), 1219-1230 (2002)

13. Mounier, H.: Propriétés structurelles des systèmes linéaires à retards : aspects théoriques et pratiques. Thèse de Doctorat, Université Paris-Sud, Orsay (1995)

14. Mounier, H.: Algebraic interpretations of the spectral controllability of a linear delay system. Forum Math. 10, 39-58 (1998)

15. Rodino, L.: Linear Partial Differential Operators in Gevrey Spaces. World Scientific, Singapore (1993)

16. Rudolph, J.: Flatness based control of distributed parameter systems. Berichte aus der Steuerungs- und Regelungstechnik. Shaker Verlag, Aachen (2003)

17. Rudolph, J., Winkler, J., Woittennek, F.: Flatness based control of distributed parameter systems: Examples and computer exercises from various technological domains. Berichte aus der Steuerungs- und Regelungstechnik. Shaker Verlag, Aachen (2003)

18. Rudolph, J., Woittennek, F.: Motion planning and open loop control design for linear distributed parameter systems with lumped controls. Internat. J. Control 81(3), 457-474 (2008)

19. Woittennek, F.: Beiträge zum Steuerungsentwurf für lineare, örtlich verteilte Systeme mit konzentrierten Stelleingriffen. Berichte aus der Steuerungs- und Regelungstechnik. Shaker Verlag, Aachen (2007)

20. Woittennek, F., Mounier, H.: Controllability of networks of spatially one-dimensional second order p.d.e. - an algebraic approach. SIAM J. Control Optim. 48(6), 3882-3902 (2010) 